\title{
COVID-19 Impfung: Die menschliche Doppelnatur
}

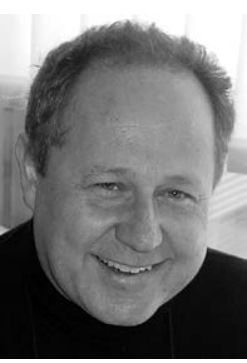

Manfred Wildner

\author{
Bibliografie \\ Gesundheitswesen 2021; 83: 83-85 \\ DOI 10.1055/a-1347-8297 \\ ISSN 0941-3790 \\ (C) 2021. Thieme. All rights reserved. \\ Georg Thieme Verlag KG, Rüdigerstraße 14, \\ 70469 Stuttgart, Germany \\ Korrespondenzadresse \\ Prof. Dr. med. Manfred Wildner \\ Pettenkofer School of Public Health \\ c/o Bayerisches Landesamt für Gesundheit und \\ Lebensmittelsicherheit \\ Veterinärstraße 2 \\ 85764 Oberschleißheim \\ Deutschland \\ Manfred.Wildner@Igl.bayern.de
}

Die Vorstellung einer Doppelnatur des Menschen findet sich schon in früher geschichtlicher und davor wohl auch vorgeschichtlicher Zeit. Teilweise wird sie philosophisch ausgedeutet als die Wesensverschiedenheit von Leib und Seele, Trieb und Vernunft, teilweise spiegelt sie sich in Erzählungen und Mythen. Bezogen auf den Mythos des Asklepios (lat. Äskulap), den altgriechischen Schutzgott der Heilkunst, findet sich diese Doppelnatur gleich zweifach. Zum einen drückt sie sich in Äskulaps Dasein als Halbgott aus, mithin als göttlich-menschliches Mischwesen, zum anderen spiegelt sie sich in dem Mischwesen, welches ihn bei sich in Obhut nahm: Cheiron. Der wegen seiner Weisheit und Gerechtigkeit hochgerühmte Kentauer, selbst Mischwesen in Pferde- und Menschengestalt, unterrichtete den schon bei Geburt verwaisten Halbgott in der Heilkunst seines göttlichen Vaters Apollo. In dieser bildhaften Sprache dürfte sich unter anderem die Einsicht um die biografischen Brüche und unterschiedlichen Seiten unseres Menschseins spiegeln: Die biologisch-triebhafte Basis ebenso wie eine diese übersteigende Fähigkeit zu Rationalität und Einsicht. Dass die Trennung zwischen Biologie und Geist bei näherer Betrachtung selbst wiederum nur vorläufig ist, ist eine Erkenntnis moderner Ethologie und neurobiologischer Hirnforschung und daran anknüpfender Analysen zur sog. Philosophie des Geistes. Für die ärztliche Praxis und ebenso für eine Tätigkeit im Bereich Public Health/Öffentliche Gesundheit bleibt sie jedoch hilfreicher Bezugs- und Ausgangspunkt, um so von den Widersprüchlichkeiten menschlicher Existenz in Gesundheit und Leid nicht allzu überrascht zu werden und sie auch als solche annehmen zu können.

Wie drückt sich diese Doppelnatur heute aus? Hier sei als Beispiel das Thema Impfen aufgegriffen, konkret zu Zeiten der CoronaPandemie der Jahre 2020/21. Merk- und denkwürdige Risse ziehen sich dabei durch die aufgeklärten Gesellschaften der westlichen Kulturen. Wissenschaftlichkeit kämpft gegen Pseudowissenschaft- lichkeit, Wissen mit Halbwissen, ernsthafte Abwägung mit leichtfertigem Denialismus, politische Zwänge mit fachwissenschaftlicher Differenzierung. Während einerseits Katastrophenfall, gesellschaftlicher Lockdown und das Schließen von Gastronomie und Kultureinrichtungen als wirtschaftlicher Shutdown zur Vermeidung exponentiell wachsender Fallzahlen zum Einsatz kommen, beklagen andere Gruppen eine vermeintliche Überreaktion bis hin zur Verleugnung der Existenz einer gesundheitlichen Notlage. Dass gezieltere Infektionsschutzmaßnahmen auf Basis differenzierter Analysen und belegter Wirksamkeit als quasi chirurgisch präzise Eingriffe mit begrenzten gesellschaftlichen Belastungen die präferierte Option wäre, ist zunächst eine kontrafaktische Überlegung. Da diese eine kluge und vorausschauende Forschungs- und Strukturpolitik und mittel- und langfristige auch entsprechende Investitionen im Bereich öffentlicher Gesundheit erfordert, wird vorerst wie so oft die Stimme fachlich-wissenschaftlicher Vernunft vergleichsweise leise bleiben müssen, den Göttern sei's geklagt. Billig und einfach ist Gesundheit eben nicht zu haben, weder in der Individualmedizin noch im Bereich der öffentlichen Gesundheit, einem schmerzhaft und chronisch unterfinanzierten Bereich.

Im weiteren Verlauf geben zudem die unvermeidliche Knappheit zu Beginn der Impfstoffverfügbarkeit und anfängliche Pannen bei durchaus herausfordernder Logistik vordergründigen Anlass zu Kritik und in der Sache größtenteils ungerechtfertigter Schuldzuweisungen. Zu möglichen wenig rationalen Beweggründen für solche Kritik wie existenzieller Angst oder sozialem Impfneid sind die weiteren Forschungsergebnisse abzuwarten (siehe auch das COSMO Snapshot monitoring [1]). Und während anfänglich noch die Diskussionen um Priorisierungen unter Bedingungen von Knappheit im Vordergrund stehen, ist absehbar, dass bald darauf die Diskussionen um Risiken und Nebenwirkungen, individuelle Freiheiten und gesellschaftliche Solidarität und um die möglichen 
Impfziele des Schutzes der Hochrisikogruppen, der Abschwächung des pandemischen Verlaufs und der effektiven Eindämmung gegenüber einer Herdenimmunität als für sich stehende Alternativen folgen werden. Im Anschluss daran wird dann möglicherweise in einer dritten Phase, wenn alle reservierten Kontingente verfügbar sind, so mancher vorbestellte und mit Bitterkeit eingeforderte Impfstoff auf unbestimmte Zeit in den Tiefkühlschränken liegen bleiben bzw. seinen verschlungenen Weg in weniger begüterte Gesellschaften finden. Womit die Pandemie zur Episode wird, bis zur nächsten Runde im Karussell des Lebens.

Die eng mit den Pockenepidemien verknüpfte Geschichte des Impfens ist dabei durchaus bunt. Wohl schon im zweiten vorchristlichen Jahrtausend war die Variolation (lat. Variola - die Pocken) in Asien bekannt und wurde als Exposition gegenüber kleineren Erregermengen der Pocken durch Schnupfen, Inhalation oder über die angeritzte Haut praktiziert. Um die erste nachchristliche Jahrtausendwende wurde sie auch vom persischen Universalgelehrten Avicenna (Ibn Sina) beschrieben. Die Technik einer aktiven Schutzimpfung mit Ausbildung einer bleibenden Immunität blieb in Europa zunächst unbeachtet und wurde erst durch die Schriftstellerin Lady Mary Montagu in England popularisiert. Sie hatte 1718 in ihren Briefen aus Konstantinopel darüber berichtet, dort ihren Sohn und nach ihrer Rückkehr nach England im Jahr 1721 auch ihre Tochter auf diese Weise impfen lassen - unter Aufsicht u. a. des Vorsitzenden des Royal College of Physicians und anderer Hofärzte. Nachdem im gleichen Jahr auch noch sechs Strafgefangene gegen das Angebot einer Amnestie und sechs Waisenkinder ohne bleibenden Schaden geimpft worden waren, wurde die Methode von König Georg I. allgemein gebilligt. Die Methode fand in England und später auch in Nordamerika breiten Zuspruch, nicht zuletzt auch mit Anwendungen zu militärmedizinischen Zwecken. Auf dem europäischen Kontinent blieb sie zunächst umstritten. Nachdem der englische Arzt Edward Jenner die mildere und risikoärmere Vakzination mit Kuhpocken (lat. Vacca - die Kuh), wie schon andere Ärzte vor ihm, im Jahr 1796 mit Erfolg ausprobiert hatte, ersetzte sie zunehmend die Variolation und verbreitete sich auch auf dem europäischen Festland. Einer der Vorkämpfer in Deutschland war der Hannoveraner Hofmedicus Johann Christian Ballhorn, der Jenners Publikation schon ein Jahr nach ihrem Erscheinen 1798 ins Deutsche übersetzte und popularisierte [2]. Die Keimtheorie zur Ätiologie der Infektionskrankheiten blieb dabei zunächst unbeachtet, sie wurde erst im späteren 19. Jahrhundert durch Louis Pasteur zur Geltung gebracht. Im weiteren Verlauf wurde im neuen Königreich Bayern 1807 als erstem Land weltweit eine Impfpflicht - gegen die Pocken - eingeführt, andere Länder folgten. Im Deutschen Reich wurde die Pockenimpfung 1874 verpflichtend und ein Jahrhundert später, 1976 nach erfolgreicher weltweiter Eradikation der Pocken, in unserer heutigen Bundesrepublik aufgehoben.

Doch auch Misserfolge und unerfüllte Hoffnungen bzw. unerwartete Nebenwirkungen blieben nicht aus. Zu nennen sind noch im 19. Jahrhundert Robert Kochs erfolglose Tuberkuloseimpfung mittels Tuberkulin, im 20. Jahrhundert z. B. die gehäuften Fälle von Guillain-Barrée-Syndrom (GBS) nach den Schutzimpfungen gegen Schweingrippe 1976/77 in den USA und das Auftreten von Darmverschlüssen bei Kindern 1998 bei erstmaliger Einführung eines Rotavirusimpfstoffes, woraufhin die Impfstoffe wieder vom Markt genommen werden mussten. Hinzu kommen sehr seltene Neben- wirkungen verschiedener handelsüblicher Impfstoffe, welche in einer Nutzen-Risikoabwägung wegen des weitaus überwiegenden Nutzens in Kauf genommen werden [3,4]. Aktuelle und noch nicht vollständig geklärte Fragen betreffen darüber hinaus die Thematiken Narkolepsie nach Impfungen gegen die Neue Grippe 2009 [5] oder die eines Antibody-dependent Enhancement (ADE) bei Zweitinfektionen mit SARS-CoV-2 [6]. Manche vermeintliche Impfnebenwirkung ist auch schlicht eine interessensgeleitete Falschbehauptung [7], psychologische Faktoren sind von erheblicher Bedeutung [8].

Welche Lehren lassen sich daraus für eine Impfkampagne, die mehr ist als eine Summe von Angeboten der Einzelimpfung, gewinnen? Gerade für den überlegten, evidenzorientierten Ansatz einer Impfkampagne ist mit der menschlichen Doppelnatur zu rechnen. Der biomedizinische „leibliche“ Ansatz verlangt eine sorgfältige, empirische Evaluation der Impfeffektivität in den verschiedenen Alters- und Zielgruppen und für die unterschiedlichen Impfprinzipien und -strategien: Welcher Anteil an Erkrankungen lässt sich verhindern und lässt sich darüber hinaus eine sterile Immunität erreichen, das heißt eine Weitergabe des Virus durch die geimpften Personen unterdrücken? Welche Risiken und Nebenwirkungen treten auf - kurz-, mittel- und langfristig und wie sind diese gegenüber den Risiken einer Erkrankung zu gewichten? Lassen sich überhaupt schon Aussagen zu Langzeiteffekten treffen und falls ja, mit welchen Limitationen? Wie sieht es mit sehr seltenen Nebenwirkungen aus, die gleichwohl mit sehr großen Impfzahlen politisch brisant werden können? Wie lassen sich solche Überlegungen in eine konkrete Impfstrategie übersetzen - sollte vor diesem Hintergrund vielleicht bewusst nur eine Impfung von besonderen Risikogruppen mit eindeutig positivem Nutzen-Risikoverhältnis forciert werden? Sollte eine auf Fremdnützigkeit ausgerichtete Herdenimmunität überhaupt angestrebt werden, womöglich unter Einbeziehung von Kindern und Jugendlichen, solange nicht umfassende Daten zu langfristigen Risiken gewonnen sind?

Gleichzeitig ist auch die Diskussion zu führen, welche in Ergänzung zur biomedizinischen Perspektive das menschliche Sozial- und Geistesleben als zweite Natur angeht: Was ängstigt die Einzelnen, welche Diskurse und Narrative prägen Denken und Einstellungen [9]? Welche individuellen und gesellschaftlichen Freiheitsrechte sind mit dem biomedizinisch sinnvollen Vorgehen in Balance zu bringen? Wieviel Solidarität schulden wir uns gegenseitig und welche Form der Solidarität darf nur als Akt der Freiheit angesprochen werden? Wo endet eine immer auch subjektive Diskussion über Vernunft und Unvernunft - vernünftig ist immer nur der oder die, welche meinen Standpunkt teilt? Verantwortungsvolle politische Entscheidungen sind darüber hinaus durch Evidenz immer nur zu informieren. Sie enthalten nicht nur die Abwägung „richtig/falsch“, sondern auch Abwägungen zur sozialen Akzeptanz, zur Nachhaltigkeit und Anschlussfähigkeit an andere Maßnahmen, zum (verfassungs)rechtlich Erlaubten und gesellschaftlich Erwünschten, nicht zuletzt auch zu den ökonomischen Opportunitätskosten. Auch wenn das Infektionsschutzgesetz die Einschränkung von Grundfreiheiten angesichts des Höchstwertes des menschlichen Lebens erlaubt - sie ist keine „Carte blanche“, Würde und Freiheit sind Teil dieses Höchstwertes und stehen in der Güterabwägung i.d.R. noch über der Gesundheit. Auch sind politische und administrative Entscheidungen oft auch nur unter Zeitdruck und Unsicher- 
heit zu treffen und bedingen Abstriche bei mancher fachlich wünschenswerten, aber zeitlich nicht einlösbaren Präzisierung [10]. Die Anforderungen an einen gelingenden Umgang mit der strategischen Handlungs- und Krisenbewältigungsoption Impfen sind somit im 21. Jahrhundert komplex. Sie verlangen eine dieser Komplexität und auch der menschlichen Daseinsweise entsprechende breite, biomedizinische und sozial- und geisteswissenschaftliche Kompetenz. Gleichzeitig sind sie mit den Mitteln des 21. Jahrhunderts fachlich disziplinär und transdisziplinär auf eine Weise bearbeitbar, die früheren Zeiten verschlossen war.

Die Offenheit des wissenschaftlichen Diskurses bleibt daher auch beim Impfen eine elementare Voraussetzung für erfolgreiche Impfkampagnen, auf der Ebene der Biomedizin wie auf der Ebene der gesellschaftlichen Vernunft. In diesem offenen wissenschaftlichen Sinn wollen auch die Beiträge dieser Ausgabe wieder ganz allgemein einen Beitrag leisten: Die Themen sind die Frage nach einer erfolgreichen Strategie gegen den drohenden Landärztemangel, Erfahrungen mit der Delegation von Hausbesuchen und Qualifikation nicht-ärztlicher Mitarbeiter in sächsischen Hausarztpraxen, eine gelingende interprofessionelle Versorgung von Patienten mit rheumatoider Arthritis aus hausärztlicher Sicht, Prädiktoren der Inanspruchnahme von kardiovaskulären und respiratorischen Notfallaufnahmen, Referenzwerte für die kardiorespiratorische Fitness der allgemeinen Bevölkerung aus der Studie zur Gesundheit Erwachsener in Deutschland (DEGS1), die Vereinbarkeit von Beruf und Pflege und die Auswirkungen der Erwerbstätigkeit auf die Gesundheit pflegender Angehöriger, ein Bericht zum Brandenburger Netzwerk Gesunde Kinder sowie Prädiktoren der Teilnahmebereitschaft von Jugendlichen in Langzeitstudien aus der Ohrkan-Studie zur Freizeitlärmexposition.

Um am Ende noch einmal den Anfang aufzugreifen: Um den eingangs angesprochen mythischen Arzt Asklepios spinnen sich noch vielerlei weitere Erzählungen. So werden ihm u. a. 2 Töchter zugeschrieben, Hygieia und Panakeia. Zu Hygieia, in deren göttliche Zuständigkeit Maß und gesunde Lebensführung fallen, hat uns der Maler Gustav Klimt ein beeindruckendes Gemälde geschenkt: Es zeigt die schöne Göttin, welche aus einem Füllhorn ihre Wohltaten ausschüttet - die Früchte eines klug und verantwortlich geführten Lebens. Zu Panakeia fehlt ein Bild. In ihre göttliche Zuständigkeit sind die Heilmittel bei Krankheiten gegeben - die sprichwörtliche Bezeichnung Panazee für ein mythisches Allheilmittel ist ihrem Namen entlehnt (und vize versa). Allerdings war es mit dieser Tochter nicht so einfach, da sie häufig nur schwer aufzufinden war. Eine Deutung für das 21. Jahrhundert? Über eine gefundene wirksame und nebenwirkungsarme Impfung als Heilmittel gegen COVID-19 sollten und dürfen wir uns freuen, die (Ordnungs-)Lehren der Hygieia sollten wir trotzdem beherzigen - sie sind anders als Panakei- as medikamentöser Beistand auch kurzfristig verlässlich verfügbar. In einem gelingenden dialektischen Schritt könnte aus dieser vielschichtigen Auseinandersetzung mit scheinbar unüberbrückbaren Gegensätzen darüber hinaus ein großer Schritt in Richtung eines ökosystemischen One Health-Ansatzes folgen [11] - als Beitrag zu einer nachhaltig verträglichen Koexistenz menschlichen Lebens mit anderen das menschliche Leben tragenden Lebensformen, als „Leben, das leben will inmitten von Leben, das Leben will“ (Albert Schweitzer).

\section{Interessenkonflikt}

Die Autorinnen/Autoren geben an, dass kein Interessenkonflikt besteht.

Literatur

[1] COVID-19 Snapshot Monitoring (COSMO). URL: COVID-19 Snapshot Monitoring (COSMO) (uni-erfurt.de) accessed 12.01.2021

[2] Jenner $E$. An inquiry into the causes and effects of variolae vaccinae, a disease discovered in some western counties of England. London, Sampson Low 1798

[3] Dittmann S. Risiko des Impfens und das noch größere Risiko, nicht geimpft zu sein. Bundesgesundheitsbl 2002; 45: 316-322

[4] Oberle D, Mentzer D, Rocha F et al. Impfkomplikationen und der Umgang mit Verdachtsfällen. Bundesgesundheitsbl 2019; 62: 450-461

[5] Sarkanen TO, Alakuijala APE, Dauvilliers YA et al. Incidence of narcolepsy after $\mathrm{H} 1 \mathrm{~N} 1$ influenza and vaccinations: Systematic review and meta-analysis. Sleep Med Rev 2018; 38: 177-186

[6] Arvin AM, Fink K, Schmid MA et al. A perspective on potential antibody-dependent enhancement of SARS-CoV-2. Nature 2020; 584 : 353-363

[7] Dyer C. Lancet retracts Wakefield's MMR paper. BM] 2010; 340: c696. URL: https://doi.org/10.1136/ bmj.c696 accessed 12.01.2021

[8] Betsch C, Schmid P, Korn L et al. Impfverhalten psychologisch erklären, messen und verändern. Bundesgesundheitsbl 2019; 62: 400-409

[9] Lewandowsky S, Cook J, Schmid P et al. The COVID-19 Vaccine Communication Handbook. 2021. URL https://sks.to/c19vax, accessed 10-01-2021

[10] Whitty CJM. What makes an academic paper useful for health policy? BMC Medicine (2015); 13: 301. URL: https://bmcmedicine.biomedcentral.com/articles/10.1186/s12916-015-0544-8 accessed 10.01.2021

[11] Taking a Multisectoral, One Health Approach: A Tripartite Guide to Addressing Zoonotic Diseases in Countries. FAO/OiE/WHO, Genf 2019. URL: https://www.who.int/initiatives/tripartite-zoonosis-guide accessed 10.01.2021 